\title{
Platelet mitochondrial DNA methylation predicts future cardiovascular outcome in adults with overweight and obesity
}

Sarah Corsi ${ }^{1}$, Simona lodice ${ }^{2}$, Luisella Vigna ${ }^{3}$, Akin Cayir ${ }^{4}$, John C. Mathers ${ }^{1}$, Valentina Bollati ${ }^{2}$ and Hyang-Min Byun ${ }^{*}$ (D)

\begin{abstract}
Background: The association between obesity and cardiovascular disease (CVD) is proven, but why some adults with obesity develop CVD while others remain disease-free is poorly understood. Here, we investigated whether mitochondrial DNA (mtDNA) methylation in platelets is altered prior to CVD development in a population of adults with overweight and obesity.

Methods: We devised a nested case-control study of 200 adults with overweight or obesity who were CVD-free at baseline, of whom 84 developed CVD within 5 years, while 116 remained CVD-free. Platelet mtDNA was isolated from plasma samples at baseline, and mtDNA methylation was quantified in mitochondrially encoded cytochromeC-oxidase I (MT-CO1; nt6797 and nt6807), II (MT-CO2; nt8113 and nt8117), and III (MT-CO3; nt9444 and nt9449); tRNA leucine 1 (MT-TL1; nt3247 and nt3254); D-loop (nt16383); tRNA phenylalanine (MT-TF; nt624); and light-strand-originof-replication (MT-OLR; nt5737, nt5740, and nt5743) by bisulfite-pyrosequencing. Logistic regression was used to estimate the contribution of mtDNA methylation to future CVD risk. ROC curve analysis was used to identify the optimal mtDNA methylation threshold for future CVD risk prediction. A model was generated incorporating methylation at three loci (score 0, 1, or 2 according to 0, 1, or 2-3 hypermethylated loci, respectively), adjusted for potential confounders, such as diastolic and systolic blood pressure, fasting blood glucose, and cholesterol ratio. mtDNA methylation at MT-CO1 nt6807 (OR $=1.08,95 \% \mathrm{Cl} 1.02-1.16 ; P=0.014)$, MT-CO3 nt9444 (OR $=1.22,95 \% \mathrm{Cl}$ 1.02-1.46, $P=0.042)$, and MT-TL1 nt3254 (OR = 1.30, 95\% Cl 1.05-1.61, $P=0.008)$ was higher at baseline in those who developed CVD by follow-up, compared with those who remained CVD-free. Combined use of the three loci significantly enhanced risk prediction, with hazard ratios of $1.38(95 \% \mathrm{Cl} 0.68-2.78)$ and $2.68(95 \% \mathrm{Cl} 1.41-5.08)$ for individuals with score 1 or 2 , respectively $(P=0.003)$. Methylation at these sites was independent of conventional CVD risk factors, including inflammation markers, fasting blood glucose concentration, and blood pressure.

Conclusions: Methylations of MT-CO1, MT-CO3, and MT-TL1 are, together, strong predictors of future CVD incidence. Since methylation of these mtDNA domains was independent of conventional CVD risk factors, these markers may represent a novel intrinsic predictor of CVD risk in adults with overweight and obesity.
\end{abstract}

Keywords: mtDNA, DNA methylation, Platelets, CVD, Obesity

\footnotetext{
* Correspondence: Hyang-min.byun@newcastle.ac.uk

${ }^{1}$ William Leech Building, Population Health Sciences Institute, Newcastle

University, Newcastle upon Tyne NE2 4HH, UK

Full list of author information is available at the end of the article
}

(c) The Author(s). 2020 Open Access This article is distributed under the terms of the Creative Commons Attribution 4.0 International License (http://creativecommons.org/licenses/by/4.0/), which permits unrestricted use, distribution, and

reproduction in any medium, provided you give appropriate credit to the original author(s) and the source, provide a link to the Creative Commons license, and indicate if changes were made. The Creative Commons Public Domain Dedication waiver (http://creativecommons.org/publicdomain/zero/1.0/) applies to the data made available in this article, unless otherwise stated. 


\section{Background}

Cardiovascular disease (CVD) is the single largest cause of death and is responsible for approximately $30 \%$ of all deaths worldwide [1]. Overweight and obesity are risk factors for CVD, attributed to insulin resistance [2], inflammation [3-5], and the hyperaggregability of platelets [6]. Subsequently, inflammation markers, such as C-reactive protein (CRP) [7], uric acid (UA) [8, 9], and fibrinogen [10], are used for CVD risk prediction, as are markers of platelet activation such as lipoproteinassociated phospholipase A2 (Lp-PLA2) [11]. However, not everyone with obesity develops CVD, and the reasons why some individuals with obesity develop CVD while others remain CVD-free are poorly understood.

Mitochondrial dysfunction and damage have been implicated in obesity $[12,13]$ and CVD [14]. In particular, platelet mitochondria are important in maintaining thrombosis and hemostasis [15]. Intriguingly, platelets show hyperaggregability in adults with obesity and are unresponsive to anticoagulant treatment $[6,16]$. Mitochondria contain a circular genome of approximately $17 \mathrm{~kb}$ in size with 37 genes encoding for proteins, ribosomal RNAs, and transfer RNAs related to oxidative phosphorylation. There is growing evidence for epigenetic regulation of mitochondrially encoded genes through DNA methylation, supported by the identification of DNA methyltransferase activity in mitochondria [17], and these epigenetic marks are altered in response to environmental exposures $[18,19]$ and in disease states such as cancer [20]. It has recently been demonstrated that mitochondrial DNA (mtDNA) in platelets is aberrantly methylated in CVD patients [21], but whether this precedes disease development is not known. Supporting the hypothesis that such epigenetic changes in the mitochondrial epigenome may be early events related to CVD development, nuclear DNA methylation patterns in the liver are known to be modified by obesity [22], while epigenetic analysis of blood samples predicts future CVD risk [23-26]. DNA methylation is not only modified in CVD patients [27, 28], but also with exposure to CVD risk factors [29-33]. Further, the associations between DNA methylation and CVD events are often stronger in individuals with pre-existing CVD risk markers, such as obesity [23, 34]. Therefore, we hypothesized that aberrant platelet mtDNA methylation occurs in at-risk individuals, such as adults with obesity, prior to developing CVD and may therefore serve as a biomarker of CVD risk.

Here, we tested this hypothesis in a nested casecontrol study investigating the utility of platelet mtDNA methylation to predict future CVD events in adults with overweight or obesity who were CVD-free at baseline.

\section{Results \\ Characteristics of participants}

The mean age of participants $(n=200)$ was 62 years $(\mathrm{SD}=10)$, and $61 \%(n=122)$ were female. The participants were overweight or obese (mean BMI = 35.5, SD = 5.1) and without CVD diagnosis at baseline. These participants were followed for up to 5 years, and the incidence of CVD was recorded (Fig. 1). At baseline, those participants who developed CVD during follow-up were BMI- and sex-matched to those who remained CVD-free. In addition, smoking status, education levels, blood pressure (systolic and diastolic (SBP and DBP)), fasting blood glucose, total cholesterol, high-density lipoprotein (HDL), lowdensity lipoprotein (LDL), and triglyceride levels at baseline were not significantly different by future CVD status $(P>0.05)$ (Table 1$)$. Total cholesterol to HDL cholesterol ratio (TC/HDL) was lower at baseline in those who remained CVD-free compared to participants who developed CVD (CVD-free: mean = 3.7, $\mathrm{SD}=1.1$; CVD-developed: mean $=3.9, \mathrm{SD}=1.2$; $P=0.039$ ) (Table 1).

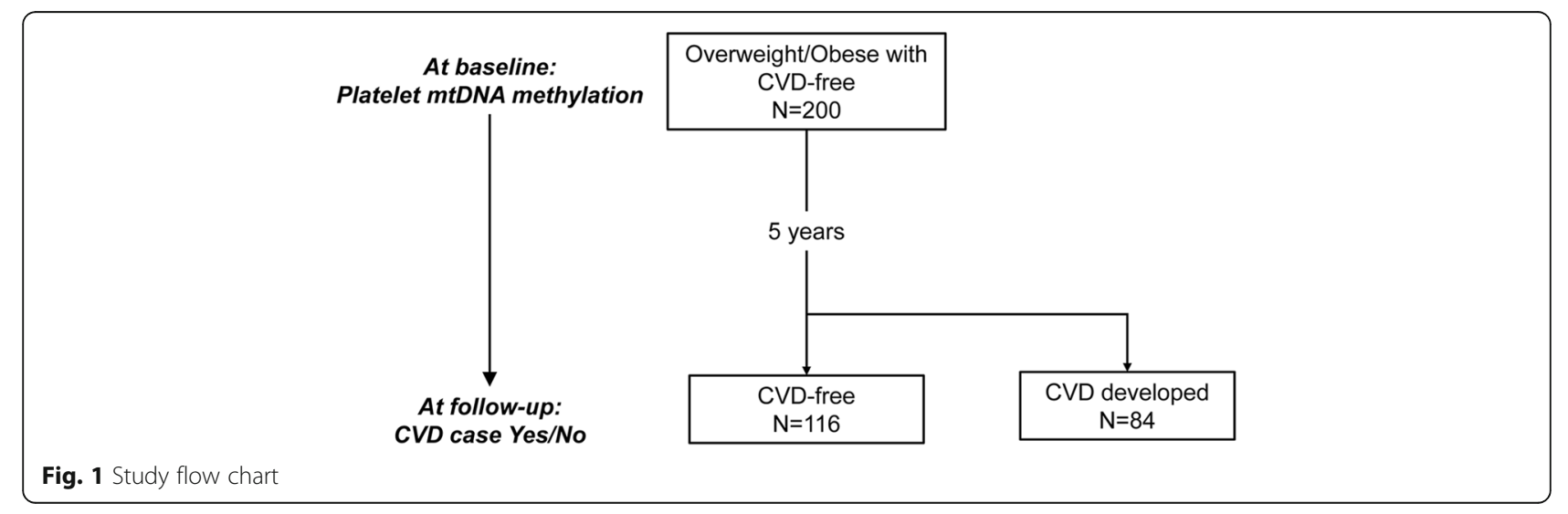


Table 1 Participant characteristics at baseline

\begin{tabular}{|c|c|c|c|c|}
\hline Variable & $\begin{array}{l}\text { All participants } \\
(n=200)\end{array}$ & $\begin{array}{l}\text { CVD-free at the } \\
\text { follow-up }(n=116)\end{array}$ & $\begin{array}{l}\text { CVD-developed at the } \\
\text { follow-up }(n=84)\end{array}$ & $P$ value \\
\hline \multicolumn{5}{|l|}{$\operatorname{Sex}(n, \%)$} \\
\hline Male & 78 (39\%) & $44(38 \%)$ & $34(40 \%)$ & \multirow[t]{2}{*}{0.716} \\
\hline Female & $122(61 \%)$ & $72(62 \%)$ & $50(60 \%)$ & \\
\hline Age (mean, SD) & $62.5, \pm 10$ & $61.7, \pm 9.5$ & $63.5, \pm 10.6$ & 0.210 \\
\hline BMI (mean, SD) & $35.5, \pm 5.1$ & $35.4, \pm 4.9$ & $35.5, \pm 5.4$ & 0.936 \\
\hline \multicolumn{5}{|l|}{ BMI categorical $(n, \%)$} \\
\hline 25.1-30.0 (overweight) & $34(17 \%)$ & $22(19 \%)$ & $12(14 \%)$ & \multirow[t]{3}{*}{0.762} \\
\hline 30.1-34.9 (obesity I) & $62(31 \%)$ & $33(28 \%)$ & $29(35 \%)$ & \\
\hline > 35.1 (obesity II and III) & $104(52 \%)$ & $61(53 \%)$ & $43(51 \%)$ & \\
\hline \multicolumn{5}{|l|}{ Smoking status $(n, \%)$} \\
\hline Never & $89(45 \%)$ & $53(46 \%)$ & $36(43 \%)$ & \multirow[t]{3}{*}{0.859} \\
\hline Former & $91(46 \%)$ & $50(43 \%)$ & $41(49 \%)$ & \\
\hline Current & 19 (10\%) & $13(11 \%)$ & $6(7 \%)$ & \\
\hline \multicolumn{5}{|l|}{ Education, years of education $(n, \%)$} \\
\hline Primary school and other ( $<5$ years) & $34(17 \%)$ & $18(16 \%)$ & $16(19 \%)$ & \multirow[t]{3}{*}{0.297} \\
\hline Secondary school and high school (< 13 years) & $129(65 \%)$ & $76(66 \%)$ & $53(63 \%)$ & \\
\hline University degree (> 14 years) & $32(16 \%)$ & $21(18 \%)$ & $11(13 \%)$ & \\
\hline $\mathrm{SBP}, \mathrm{mmHg}($ mean, SD) & $128.2, \pm 13.7$ & $129.1, \pm 13.3$ & $127, \pm 14.1$ & 0.268 \\
\hline $\mathrm{DBP}, \mathrm{mmHg}($ mean, SD) & $78.9, \pm 8.5$ & $79.2, \pm 8.5$ & $78.4, \pm 8.5$ & 0.517 \\
\hline Fasting blood glucose, mmol/L (mean, SD) & $5.9, \pm 1.4$ & $5.8, \pm 1.4$ & $6.0, \pm 1.4$ & 0.384 \\
\hline Total cholesterol, mg/dL (mean, SD) & $206.6, \pm 42.9$ & $204.5, \pm 42.4$ & $209.5, \pm 43.8$ & 0.421 \\
\hline HDL cholesterol, mg/dL (mean, SD) & $58.6, \pm 15.0$ & $60.0, \pm 15.5$ & $56.8, \pm 14.3$ & 0.141 \\
\hline LDL cholesterol, mg/dL (mean, SD) & $128.3, \pm 37.1$ & $127.6, \pm 36.0$ & $129.1, \pm 38.8$ & 0.777 \\
\hline Triglyceride (TC), mg/dL (mean, SD) & $126.4, \pm 61.6$ & $121.0, \pm 57.6$ & $133.7, \pm 66.2$ & 0.153 \\
\hline $\mathrm{TC} / \mathrm{HDL}$ ratio (mean, SD) & $3.7, \pm 1.1$ & $3.6, \pm 0.9$ & $3.9, \pm 1.2$ & 0.039 \\
\hline Framingham Risk Score, median (Q1, Q3) & $18.2(9.3,28.9)$ & $17.9(9.6,26.2)$ & $18.3(8.8,30.5)$ & 0.636 \\
\hline HeartScore, median (Q1, Q3) & $2.0(1.0,3.0)$ & $2.0(1.0,3.0)$ & $2.0(1.0,4.0)$ & 0.232 \\
\hline \multicolumn{5}{|l|}{ Medication usage $(n, \%)$} \\
\hline Not available & $19(9 \%)$ & $6(5 \%)$ & $13(16 \%)$ & \multirow[t]{3}{*}{0.039} \\
\hline Yes & $46(23 \%)$ & $30(26 \%)$ & $16(19 \%)$ & \\
\hline No & $135(68 \%)$ & $80(69 \%)$ & $55(65 \%)$ & \\
\hline
\end{tabular}

\section{Platelet mtDNA methylation at baseline by future CVD}

\section{development}

We analyzed 13 CpG sites distributed within 7 mitochondrial genomic regions (Fig. 2). Methylation at baseline was lower in those participants who remained CVD-free compared with those who developed CVD during follow-up at nt6807 of MT-CO1 (CVD-free: mean $=10.8 \pm 4.8 \%$; CVD-developed: mean $=12.5 \pm 4.8 \% ; P=0.014)$, nt9444 of MT-CO3 (CVD-free: mean $=0.7 \pm 2 \%$; CVDdeveloped: mean $=1.3 \pm 1.9 \% ; P=0.042$ ), and nt3254 of MT-TL1 (CVD-free: mean $=2.4 \pm 1.5 \%$; CVD-developed: mean $=3.0 \pm 1.6 \% ; P=0.008$ ) (Fig. $3 \mathrm{a}-\mathrm{c}$ ). No significant differences in methylation were present for the other $\mathrm{CpG}$ sites measured.
We then examined methylation at the three loci in relation to the development of CVD during follow-up. The odds ratios (ORs) for developing CVD during follow-up were 1.08 (95\% CI 1.02-1.16) for nt6807 of MT-CO1, 1.22 (95\% CI 1.02-1.46) for nt9444 of $M T$ CO3, and 1.30 (95\% CI 1.05-1.61) for nt3254 of $M T$ TL1, adjusted for age, BMI, fasting blood glucose, cholesterol ratio, SBP, and DBP (Fig. 4). Logistic regression demonstrated that there were no significant associations between mtDNA methylation of $M T$ CO1, MT-CO3, and MT-TL1 and conventional CVD risk biomarkers at the baseline, including insulin resistance (HOMA-IR), age, cholesterol level, serum uric acid, and BMI (Table S1). 


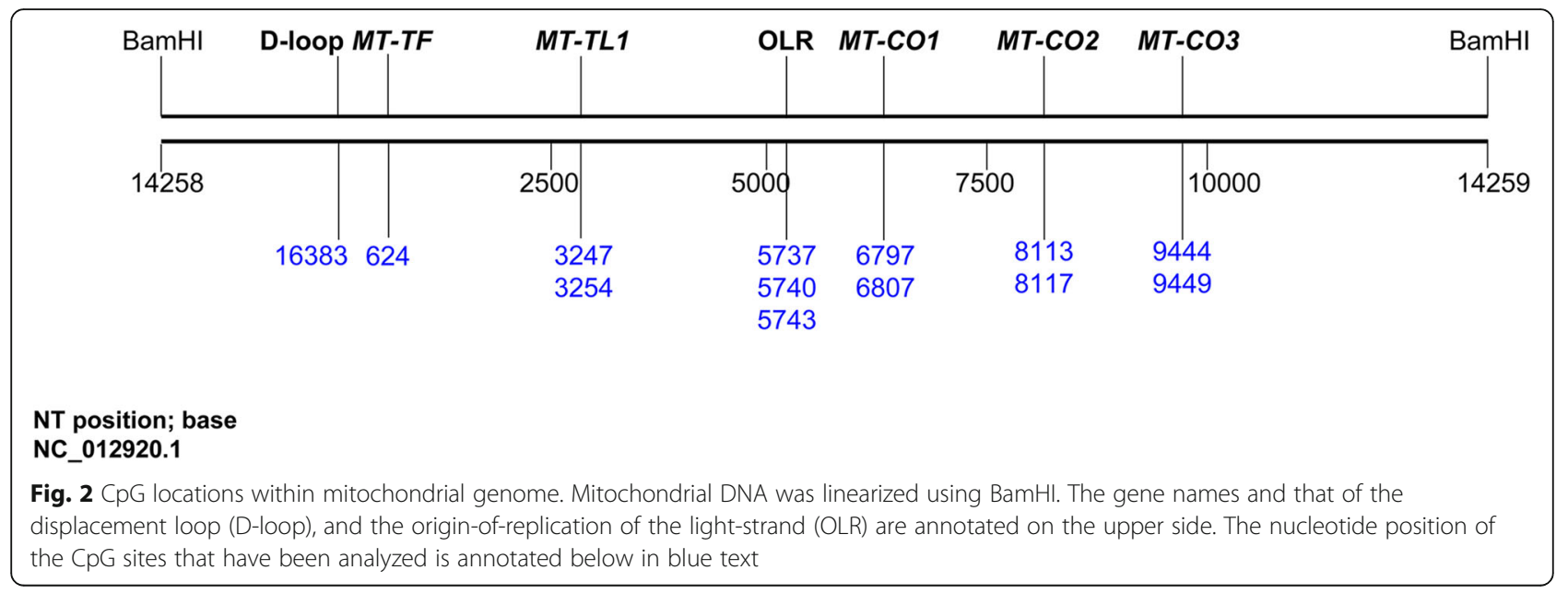

Utility of platelet mtDNA methylation to predict CVD risk Receiver-operating characteristic (ROC) curves were generated to determine the optimal threshold of mtDNA methylation (\%) for each $\mathrm{CpG}$ site at baseline to discriminate between CVD-free and CVD-developed individuals at follow-up (Fig. 5). Thresholds of $12 \%$ for MT-CO1 nt6807 $(P=0.049), 1.5 \%$ for $M T-C O 3$ nt9444 $(P=$ $0.001)$, and $3 \%$ for $M T-T L 1$ nt3254 $(P=0.22)$ yielded maximum discrimination between CVD-free and CVDdeveloped participants (Table 2 (a)). TC/HDL cholesterol, which differed between groups at baseline, was not a predictor of CVD risk during follow-up $(P=0.38)$ (Fig. 5 d, Table 2 (a)).

The threshold values that maximized sensitivity and specificity to predict CVD risk were used to create dichotomous variables "methylation level above the
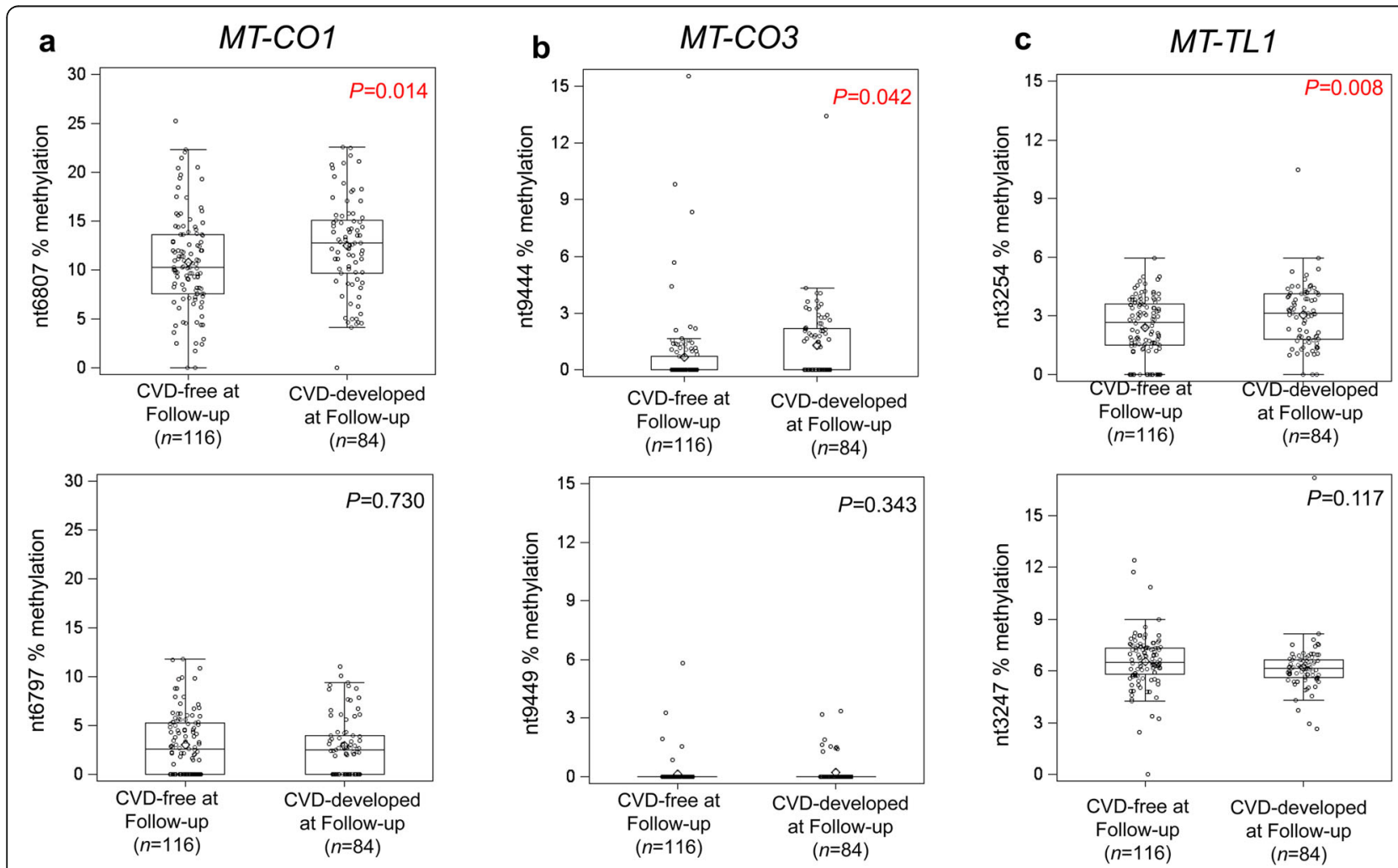

Fig. 3 Distribution of mtDNA methylation at baseline among individuals who remained CVD-free and who develop CVD during follow-up. Methylation at two CpG positions for MT-CO1 (a), MT-CO3 (b), and MT-TL1 (c), examined by pyrosequencing. The top panels report the CpG sites whose methylation significantly differs between the CDV-free and CVD-developed at follow-up. The $P$ values were calculated by $t$ test 

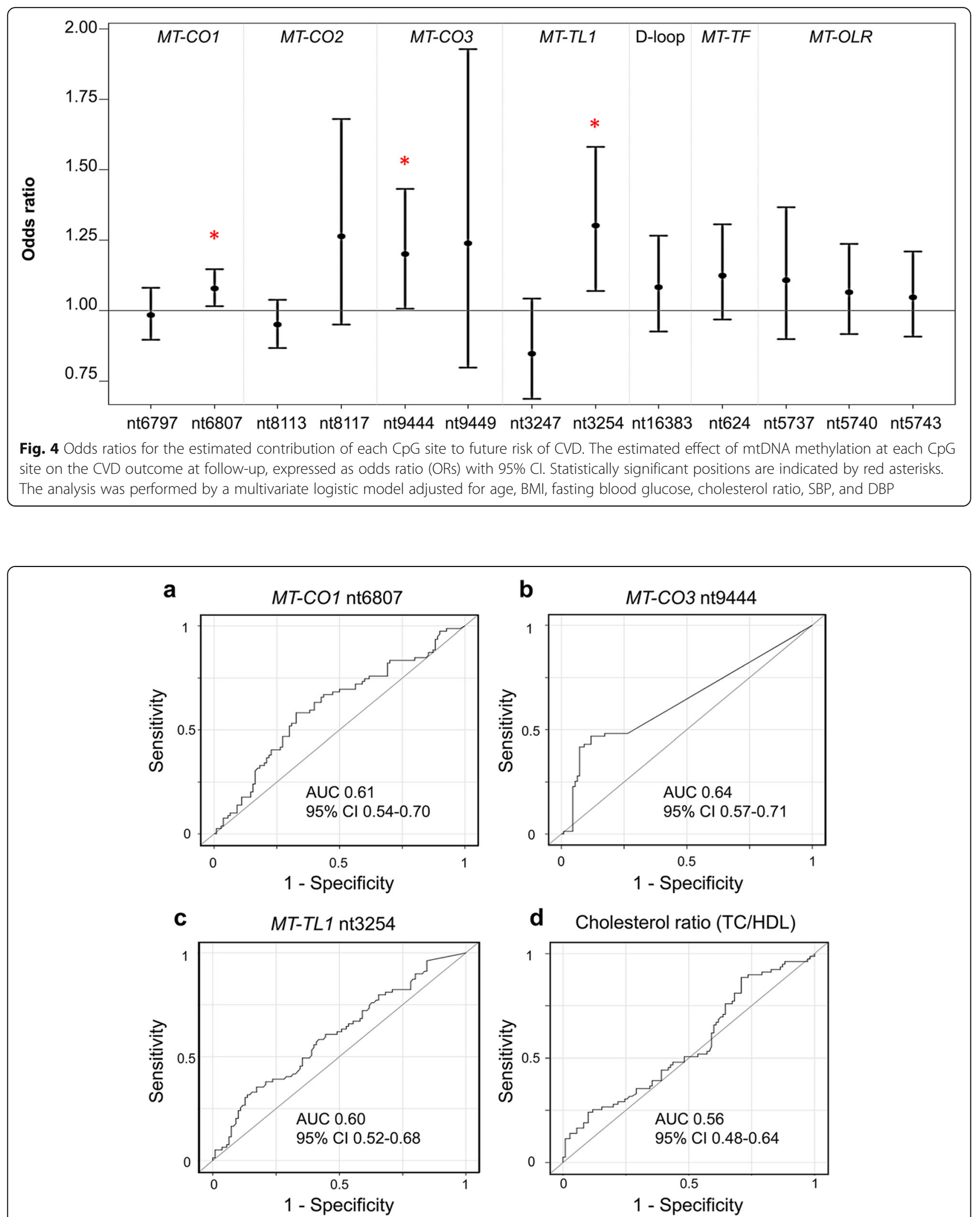

Fig. 5 ROC curves for methylation at three loci and cholesterol ratio for prediction of CVD outcome. Discrimination ability of methylation at three CpG sites (MT-CO1 nt6807, MT-CO3 nt9444, and MT-TL1 nt3254) (a-c) and the cholesterol ratio (TC/HDL) (d) to predict CVD incidence within 5 years of baseline. Area under the ROC curve (AUC) and $95 \% \mathrm{CI}$ values are annotated 
Table 2 MtDNA methylation thresholds for each CpG site and score for predicting CVD outcome

\begin{tabular}{|c|c|c|c|c|c|c|}
\hline \multicolumn{7}{|c|}{ a. Threshold for each CpG site } \\
\hline & Methylation & $\begin{array}{l}\text { Median survival } \\
\text { time (months) }^{*}\end{array}$ & At risk & $\begin{array}{l}\text { CVD during } \\
\text { follow-up }\end{array}$ & CVD free & $\begin{array}{l}\text { Log-rank } \\
P \text { value }\end{array}$ \\
\hline \multicolumn{2}{|l|}{ All patients } & 43.8 & 200 & 84 & 116 & \\
\hline \multirow{2}{*}{$\begin{array}{l}\text { MT-CO1 nt6809 } \\
\text { (\% methylation) }\end{array}$} & $<12.0$ & 47.5 & 114 & 35 & 79 & \multirow[t]{2}{*}{0.049} \\
\hline & $\geq 12.0$ & 38.3 & 83 & 47 & 36 & \\
\hline \multirow{2}{*}{$\begin{array}{l}\text { MT-CO3 nt9444 } \\
\text { (\% methylation) }\end{array}$} & $<1.5$ & 47.0 & 146 & 44 & 102 & \multirow[t]{2}{*}{0.001} \\
\hline & $\geq 1.5$ & 33.0 & 51 & 38 & 13 & \\
\hline \multirow{2}{*}{$\begin{array}{l}\text { MT-TL1 nt3254 } \\
\text { (\% methylation) }\end{array}$} & $<3.0$ & 45.7 & 105 & 37 & 68 & \multirow[t]{2}{*}{0.22} \\
\hline & $\geq 3.0$ & 42.1 & 94 & 46 & 48 & \\
\hline \multirow[t]{2}{*}{ Cholesterol ratio } & $<3.5$ & 42.1 & 102 & 43 & 59 & \multirow[t]{2}{*}{0.38} \\
\hline & $\geq 3.5$ & 45.3 & 94 & 41 & 53 & \\
\hline \multicolumn{7}{|c|}{$\begin{array}{l}\text { b. Score for predicting } \\
\text { the CVD outcome }\end{array}$} \\
\hline Score ${ }^{* *}$ & $\begin{array}{l}\text { Median survival } \\
\text { time (months) }\end{array}$ & At risk & CVD during follow-up & CVD-free & $\%$ CVD-developed at follow-up & Log-rank $P$ value \\
\hline 0 & $\sim 60$ & 61 & 13 & 48 & $21 \%$ & \multirow[t]{3}{*}{0.003} \\
\hline 1 & 54.8 & 63 & 21 & 42 & $33 \%$ & \\
\hline 2 & 35.1 & 69 & 45 & 24 & $65 \%$ & \\
\hline
\end{tabular}

(a) MtDNA methylation thresholds for each CpG site and outcomes of survival analysis. Survival analysis for the participants stratified according to the methylation score at individual loci $(M T-C O 1, M T-C O 3$, and MT-TL1) and to the cholesterol ratio. (b) Score to predict future CVD events based on methylation at MT-CO1 nt6809, MT-CO3 nt9444, and MT-TL1 nt3254.

*Median: time in months without-CVD

**Participants with score 2 (two or three CpG sites with methylation above the thresholds) had a lower median time without-CVD (35.1 months) than the participants with score 1 (54.8 months) and score 0 (the median survival time is not reached). This analysis was performed on a total of 193 participants, for whom the methylation percentage of all the three genes was available

threshold" and "methylation level below the threshold" for each of the significant CpG sites within MT-CO1, MTCO3, and MT-TL1. Using these values, overall scores were calculated for each individual participant as follows: methylation not above the thresholds at any of the three loci (score 0), methylation above the threshold at any one locus (score 1), and methylation above the threshold at any two or all three loci (score 2) (Table 2 (b)). Compared with score 0 , the hazard ratio (HR) for developing CVD for score 1 was 1.38 (95\% CI, 0.68-2.78) and for score 2 was 2.68 (95\% CI, 1.41-5.08) (Fig. 6a). During follow-up, 65\% of the individuals with score 2 developed CVD, while only $21 \%$ of individuals with score 0 developed CVD (Fig. $6 \mathrm{a}$ and Table 2 (b)). Participants with score 2 had a lower median time without-CVD (35.1 months) than participants with score 1 (54.8 months). More than half of the participants with score 0 were CVD-free at the end of the follow-up period.

\section{Comparison with existing risk prediction models}

Conventional CVD risk prediction scores were calculated at baseline using the Framingham Risk Score [35] and the European HeartScore [36]. These scores were not different between those who remained CVD-free and the CVDdeveloped group $(P=0.636$ and $P=0.232$, respectively), demonstrating the potential utility of mtDNA methylation as a predictor of CVD development.

\section{Sensitivity analysis}

A sensitivity analysis was performed by excluding participants who developed CVD within a year from baseline, but this did not change the relationships previously observed (Fig. 6b). The HR for those who scored 2 remained significantly higher than those who scored 1 $(\mathrm{HR}=2.17,95 \% \mathrm{CI} 1.06-4.47)$ and was even higher in comparison with those who scored $0(\mathrm{HR}=2.53,95 \% \mathrm{CI}$ 1.12-5.72) (Fig. 6b). An additional sensitivity analysis was performed by stratifying the CVD cases into "Mild," such as hypertension $(n=51)$, and "Severe" events, such as ischemic heart diseases $(n=33)$ (Table S2). The model was tested in the Mild subgroup and showed that the mtDNA methylation score was a significant $(P<$ 0.001) predictor of future risk of developing CVD. The HR for those who scored 2 was significantly higher than for those scored $1(\mathrm{HR}=2.27,95 \% \mathrm{CI} 1.13-4.44, P=$ $0.021)$ and those who scored 0 (HR $=4.34,95 \%$ CI $1.76-$ 10.73, $P<0.002)$. No such relationships were apparent in the Severe subgroup of CVD events $(n=33)$, due to lack of power $(P=0.086)$ (data not shown).

\section{Discussion}

To the best of our knowledge, this is the first study investigating platelet mtDNA methylation in relation to the future development of CVD. In this nested case- 

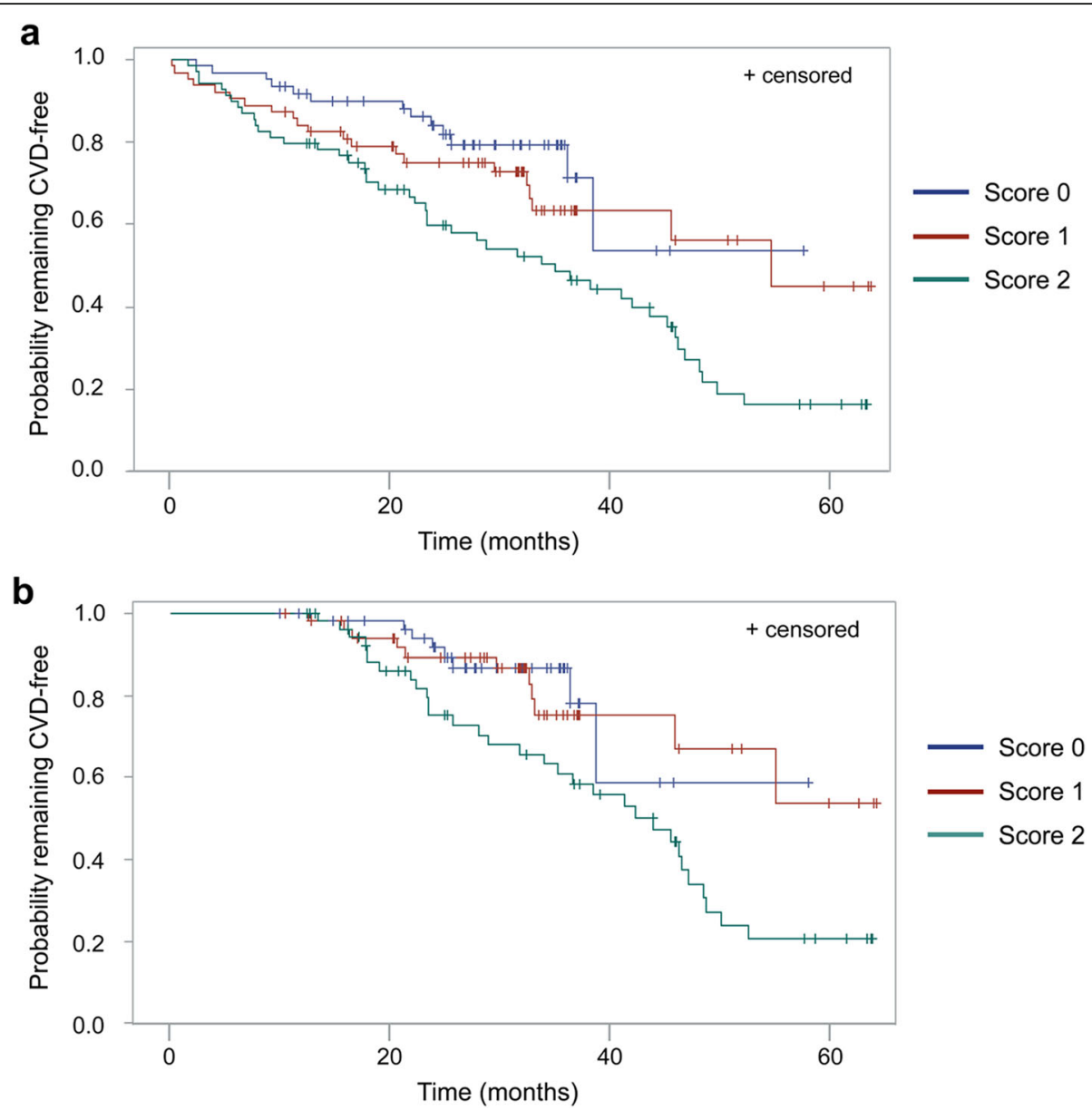

Fig. 6 The Kaplan-Meier curves for probability of remaining CVD-free by methylation score. a Time CVD-free from baseline (months) among participants categorized by methylation at MT-CO1 nt6807, MT-CO3 nt9444, and MT-TL1 nt3254. b Sensitivity analysis in which all participants who developed CVD within 1 year of baseline were excluded

control study of 200 adults with overweight and obesity, higher mtDNA methylation at three loci (MT-CO1 nt6807, MT-CO3 nt9444, and MT-TL1 nt3254) in platelets was associated with higher risk of developing CVD within 5 years. Further, participants with score 2 (high methylation at two or three loci) developed CVD significantly sooner than the participants with score 1 and score 0 . Thus, mtDNA methylation at the three loci may be a novel predictive biomarker for the future risk of developing CVD.

We have previously demonstrated changes in the mitochondrial epigenome among individuals with CVD, including hypermethylation of MT-CO1, MT-CO3, and MT-TL1 [21]. Further, we have shown that mtDNA methylation modifies the effect of particulate matter exposure and heart rate variability, a prognostic marker of CVD [37]. We have built on our previous work to demonstrate that mtDNA methylation may serve as a predictor of CVD risk among individuals with overweight and obesity. However, the field remains at a nascent stage, with little understanding of the mechanisms underpinning how mtDNA methylation levels may be implicated in the etiology of CVD and/or platelet activation. Recently, it has been demonstrated that mtDNA methylation regulates expression of mitochondrial-derived peptides (MDP) with cytoprotective function [38] suggesting that mtDNA methylation level may be indicative of the overall stress to which the cell is exposed. Additionally, in vitro studies have shown that the presence of 5methylcytosine can alter mitochondrial transcription factor (TFAM) binding and transcription initiation [39].

MtDNA methylation levels in blood are associated with blood pressure and heart rate variability in individuals with CVD-related environmental and occupational exposures [18, 19, 37, 40]. However, in platelets, we did not find any association between mtDNA methylation level and the most common CVD risk factors including age, BMI, blood pressure, blood glucose concentration, cholesterol, and uric acid in individuals with overweight and obesity. Therefore, our study supports the idea that altered mtDNA methylation in platelets precedes the development of CVD, and may serve as a non-invasive, 
easy-to-access biomarker to distinguish individuals with higher CVD risk. Adults with overweight or obesity may, therefore, benefit from identification to facilitate early primary prevention and monitoring to reduce their personal risk of CVD.

We observed low levels of mtDNA methylation in these participants and subtle, but detectable, differences between individuals who developed CVD during follow-up and those who remained CVD-free. Such subtle changes in methylation are not confined to the mitochondrial epigenome, as changes in methylation of $<5 \%$ are frequently reported in aging, in response to environmental exposures [41], and during disease initiation [42]. It is not known whether these small changes in DNA methylation reflect changes in gene expression. Regardless, they may serve as a biomarker of a cascade of other biological reactions [4345], such as MDP regulation [38].

Our study has limitations that merit consideration. The outcome in our study was diagnosis of any of a heterogeneous group of CVDs that ranged from mild (e.g., hypertension) to more severe events. Our model remained strong in predicting the "mild" CVD events, but the lack of statistical power prevented examination of its ability to predict more "severe" cases. Further, replication of our findings is imperative. Such a validation would require access to data and samples from a cohort that had collected plasma or platelets and had follow-up data on CVD incidence as part of a prospective study of individuals with overweight and obesity. We utilized hospital discharge records, which are widely used for collection of data regarding clinical diagnoses (e.g., for Italian healthcare administrative databases and the WHO's European Health Information Gateway for classification), but which can potentially under- or overestimate the number of cases. The use of thoroughly validated administrative databases may strengthen future studies. We attempted a partial validation by dividing the population of 200 individuals into a test set $(n=150)$ and a validation set $(n=50)$ with the same proportions of CVD-free and CVDdeveloped at follow-up participants in both, which showed that the mtDNA methylation markers predicted CVD risk in both the test $(P=0.045)$ and validation sets $(P=0.034)$. Finally, as most of the participants were Caucasian, additional studies are needed to validate these findings in individuals with different ethnicities.

In conclusion, we have demonstrated that mtDNA methylation of MT-CO1, MT-CO3, and MT-TL1 in platelets from adults with overweight and obesity may predict CVD risk during the following 5 years. Our findings require confirmation in a larger, independent study.

\section{Methods}

\section{Study design and sample selection}

We utilized plasma samples and clinical data from the Susceptibility to Particle Health Effects, miRNAs and
Exosomes (SPHERE) study in which 2000 participants with overweight $\left(25<\mathrm{BMI}<30 \mathrm{~kg} / \mathrm{m}^{2}\right)$ and obesity $\left(B M I \geq 30 \mathrm{~kg} / \mathrm{m}^{2}\right)$ were recruited in Milan, Italy [46]. We designed a prospective nested case-control study using samples and data from 200 participants within the SPHERE study without previous hospitalization for CVD at the time of enrolment (baseline; $n=200$ ) for whom follow-up data for up to 5 years (median $=27$ months) were available. For those who developed CVD, the follow-up stopped after the first CVD diagnosis; for those who remained CVD-free, the follow-up lasted until the last update from the Italian National Health Service. We selected 84 individuals who developed CVD in the follow-up period, and these were sex- and BMI-matched with 116 individuals who remained CVD-free. The demographic and clinical characteristics of these participants are summarized in Table 1. Ethical approval was provided by the Institutional Review Board, Fondazione IRCCS Cà Granda Ospedale Maggiore Policlinico at University of Milan. The ethnicity of the SPHERE study participants was predominantly Caucasian $(95.8 \%$ of cases) [46].

\section{Assessment of CVD risk at baseline and CVD events at follow-up}

To estimate individual CVD risk at baseline, we calculated the Framingham Risk Score which uses information on sex, age, SBP, treatment for hypertension, smoking, type 2 diabetes, HDL, and total cholesterol [35]. In addition, we used the HeartScore to predict the incidence of fatal CVD within 10 years [36, 47] using age, sex, SBP, cholesterol, HDL cholesterol, BMI, and smoking status.

Details of CVD events were obtained from the hospital discharge registry of the Italian National Health Service. A CVD event was defined as any principal or any 1 of 5 secondary diagnosis of diseases of the circulatory system (3digit ICD-9-CM codes from 390 to 459) [48]. A detailed list of the CVD events and antihypertensive medications of the participants by follow-up is summarized in the Supplementary Material (Table S2 and S3). Briefly, 47 participants (56\%) were diagnosed with hypertension and 37 participants (44\%) were diagnosed with other forms of CVD including atrial fibrillation and atherosclerosis (Table S2).

\section{Platelet mtDNA preparation and DNA methylation measurement}

Plasma samples from 200 participants were used to isolate platelet mtDNA as described previously [21]. Briefly, platelet pellets obtained by centrifugation of $200 \mu \mathrm{L}$ plasma at $1400 \times g$ were treated with DNaseI (30 U, ROCHE) to eliminate cell-free nuclear DNA containing nuclear mitochondrial DNA segments (NUMTs). The EZ DNA Methylation Direct kit (Zymo Research) was used for extraction and 
bisulfite conversion of mtDNA simultaneously. To maximize bisulfite conversion efficiency [49], mtDNA was linearized with BamHI (NEB) following proteinase $\mathrm{K}$ treatment. The bisulfite-converted mtDNA $(20 \mu \mathrm{L})$ was stored at $-80^{\circ} \mathrm{C}$ until analysis.

Bisulfite-PCR reactions were performed using $1 \mu \mathrm{L}$ of bisulfite-converted mtDNA, $9 \mu \mathrm{L}$ water, $12 \mu \mathrm{L}$ Hot-Start $\mathrm{GoTaq}^{\circ} \mathrm{DNA}$ Polymerase (Promega), $1 \mu \mathrm{L}$ forward primer (10 pmole), and $1 \mu \mathrm{L}$ reverse biotin-labeled primer (10 pmole). We investigated seven regions: mitochondrially encoded cytochrome-C-oxidases I, II, and III (MT-COI, $M T-C O 2$, and $M T-C O 3)$; mitochondrially encoded tRNA leucine 1 (MT-TL1) and tRNA phenylalanine (MT-TF); Dloop; and mitochondrially encoded light-strand origin-ofreplication $(M T-O L R)$. DNA methylation was measured at two CpG sites within MT-CO1 (nucleotide (nt) positions 6797 and 6807), MT-CO2 (nt8113 and nt8117), MT-CO3 (nt9444 and nt9449), and MT-TL1 (nt3247 and nt3254); one CpG site within D-loop (nt16383) and MT-TF (nt624); and three within MT-OLR (nt5737, nt5740, and nt5743) (Table S4). The provided mtDNA sequences and the nucleotide positions are based on NCBI reference sequence NC_012920.1. Amplified mtDNA PCR products were then used for pyrosequencing reactions (PyroMark Q96 ID, QIAGEN) as described elsewhere [18, 19, 21, 50]. Each sample was analyzed in duplicate (Pearson's correlation coefficient $=0.74$ for technical replicates; coefficient of variation $12.5 \%$ ), and the mean of replicates was used for further analysis. The correlations between methylation at different CpG sites within each gene were low (Table S5), and we therefore treated each $\mathrm{CpG}$ as a separate data point.

\section{Statistical analysis}

For normally distributed demographic and clinical characteristics and for DNA methylation levels, data are expressed as mean and standard deviation, otherwise by median and range. Frequencies and percentages were calculated for categorical variables. Data for CVD-free and CVD-developed participants at follow-up were compared using the $\chi^{2}$ test for categorical data and Student's $t$ test for continuous variables. Multivariate logistic regression, adjusted for age, BMI, fasting blood glucose, cholesterol ratio (TC/HDL), SBP, and DBP, was performed to investigate the association between DNA methylation at each locus (CpG site) and the risk of CVD during follow-up. Estimated effects were reported as ORs and 95\% confidence intervals $(\mathrm{CI})$ associated with an increase in 5methylcytosine $(5 \mathrm{mC})$ at each locus.

ROC curves were generated to evaluate the diagnostic ability of the cholesterol ratio and mtDNA loci to distinguish between participants who were CVD-free and those in whom CVD-developed at follow-up. The optimum threshold was selected by the Youden Index as the one that maximized sensitivity $(\mathrm{SE})+$ specificity $(\mathrm{SP})-1$. The area under the ROC curve (AUC) and corresponding 95\% $\mathrm{CI}, \mathrm{SE}, \mathrm{SP}$, and threshold were reported for cholesterol ratio and for the MT-CO1 nt6807, MT-CO3 nt9444, and MT-TL1 nt3254 positions. For each CpG site, a dichotomous variable was created viz. "methylation level above the threshold" for the specific locus and "methylation level below the threshold." In addition, we tested the utility of a score built as the sum of the index value $(0,1)$ for each locus (MT-CO1 nt6807, MT-CO3 nt9444, and MT-TL1 nt3254) in predicting CVD. The score has three categories: none of three loci display mtDNA methylation above threshold (score 0), any one of the three loci has mtDNA methylation above threshold (score 1), and any two or all three loci display mtDNA methylation threshold (score 2).

The Kaplan-Meier survival curves and log-rank tests were calculated by stratifying CVD cases by each locus below or above the methylation threshold. To evaluate the independent prognostic value of each single locus and of their combination on future CVD cases, we calculated hazard ratios (HRs) with Cox multivariable regression models adjusted for DBP, SBP, fasting blood glucose concentration, and cholesterol ratio. The Cox multivariable regression was performed on a total of 193 participants, for whom the methylation percentage of all the three genes was available. The same model was used to evaluate the prognostic value of cholesterol ratio, when evaluated as the predictor. The assumption of proportional hazard was checked with the $\log [\log ($ survival)] plot and by the time-dependent covariate test. Cox multivariable regression models were also used to evaluate the potential prognostic value of the Framingham Risk Score and of the European HeartScore on CVD risk.

A sensitivity analysis was performed by excluding the participants who developed CVD within a year from baseline in all Cox multivariable regression models. An additional sensitivity analysis was performed by stratifying the CVD cases into "Mild," such as hypertension $(n=51)$, and "Severe" events, such as ischemic heart diseases $(n=33)$. However, the Severe event $(n=33)$ category did not provide enough power to be tested reliably (data not shown). All reported $P$ values were two-tailed, and those less than 0.05 were considered statistically significant. Statistical analyses were performed with SAS software, version 9.4.

\section{Supplementary information}

Supplementary information accompanies this paper at https://doi.org/10. 1186/s13148-020-00825-5.

Additional file 1 Table S1. Multivariate analysis for the association between potential risk factors and mitochondrial DNA methylation. Table S2. Diagnostic and procedure codes for CVD events at Follow-up. Table S3. List of antihypertensive medication. Table S4. PCR primers and pyrosequencing assays used to analyze mtDNA methylation. Table 
S5. Correlation matrix of mtDNA methylation at CpG positions included in the study.

\section{Abbreviations}

CVD: Cardiovascular disease; DBP: Diastolic blood pressure; D-loop: Noncoding position on the mtDNA; MT: Mitochondrial (standing before a name and italics denote the mitochondrially encoded genes); MT-

CO1: Mitochondrially encoded cytochrome-C-oxidase I; MT-CO2: Mitochondrially encoded cytochrome-C-oxidase II; MT-CO3: Mitochondrially encoded cytochrome-C-oxidase III; mtDNA: Mitochondrial DNA; mtDNMT: Mitochondrial DNA-methyl-transferase; MT-OLR: Mitochondrially encoded light-strand-originof-replication: MT-TF: Mitochondrially encoded TRNA phenylalanine; MTTL1: Mitochondrially encoded TRNA leucine 1; SBP: Systolic blood pressure

\section{Acknowledgements}

We are, above all, very grateful to all the participants for their collaboration. Additionally, we thank Dr. Raquel Cacace (M.D.) for the calculation of the Framingham Score and HeartScore, Ms Laura Dioni for the sample shipment, and Mario Siervo (M.D., PhD) for the classification of diseases into "Mild" and "Severe."

\section{Authors' contributions}

HMB and VB conceived the project. LV collected the plasma samples from the participants. VB and SI designed the nested case-control study. VB blinded and shipped the samples. SC carried out the experimental work on the plasma samples. SI carried out the statistical analysis. AC provided guidance on PCR and pyrosequencing. SC wrote the manuscript with critical review, commentary, and revision from HMB and JCM. All the authors reviewed and approved the final manuscript.

\section{Funding}

This work has been supported by the NIH (NIEHS, R21ES022694 to HMB) and by the European Research Council (ERC-2011-StG 282413 to VB).

\section{Availability of data and materials}

The raw data of mtDNA methylation are available from the corresponding author on reasonable request. SAS scripts used in the study are available upon request.

\section{Ethics approval and consent to participate}

Ethical approval was provided by the Institutional Review Board, Fondazione IRCCS Cà Granda Ospedale Maggiore Policlinico at University of Milan.

\section{Consent for publication}

Not applicable.

\section{Competing interests}

The authors declare that they have no competing interests.

\section{Author details}

'William Leech Building, Population Health Sciences Institute, Newcastle University, Newcastle upon Tyne NE2 4HH, UK. ${ }^{2}$ EPIGET Lab, Department of Clinical Sciences and Community Health, Università degli Studi di Milano, via San Barnaba 8, 20122 Milan, Italy. ${ }^{3}$ Department of Preventive Medicine, Occupational Health Unit, Fondazione IRCCS Ca' Granda, Ospedale Maggiore Policlinico, Milan, Italy. ${ }^{4}$ Vocational Health College, Canakkale Onsekiz Mart University, Canakkale, Turkey

Received: 10 December 2019 Accepted: 9 February 2020

Published online: 17 February 2020

\section{References}

1. Bloom DE, Cafiero ET, Jané-Llopis E, Abrahams-Gessel S, Bloom LR, Fathima $\mathrm{S}$, et al. Global economic burden non communicable diseases. Geneva: World Economic Forum.

2. Reaven GM. Insulin resistance: the link between obesity and cardiovascular disease. Medical clinics of North America. Med Clin North Am. 2011;95:875-92.

3. Karelis AD, Faraj M, Bastard J-P, St-Pierre DH, Brochu M, Prud'homme D, et al. The metabolically healthy but obese individual presents a favorable inflammation profile. J Clin Endocrinol Metab. 2005;90:4145-50.
4. Van Gaal LF, Mertens IL, De Block CE. Mechanisms linking obesity with cardiovascular disease. Nature. 2006:444:875-80

5. Wang Z, Nakayama T. Inflammation, a link between obesity and cardiovascular disease. Mediators of Inflammation. 2010;2010:e535918.

6. Bordeaux BC, Qayyum R, Yanek LR, Vaidya D, Becker LC, Faraday N, et al. Effect of obesity on platelet reactivity and response to low-dose aspirin. Prev Cardiol. 2010;13:56-62.

7. Ridker PM, Cannon CP, Morrow D, Rifai N, Rose LM, McCabe CH, et al. Creactive protein levels and outcomes after statin therapy. N Engl J Med. 2005;352:20-8.

8. Ioachimescu AG, Brennan DM, Hoar BM, Hazen SL, Hoogwerf BJ. Serum uric acid is an independent predictor of all-cause mortality in patients at high risk of cardiovascular disease: a preventive cardiology information system (PreCIS) database cohort study. Arthritis Rheum. 2008;58:623-30.

9. Wang JG, Staessen JA, Fagard RH, Birkenhäger WH, Gong L, Liu L. Prognostic significance of serum creatinine and uric acid in older Chinese patients with isolated systolic hypertension. Hypertension. 2001;37:1069-74.

10. Appiah D, Schreiner PJ, MacLehose RF, Folsom AR. Association of plasma $\gamma^{\prime}$ fibrinogen with incident cardiovascular disease: the Atherosclerosis Risk in Communities (ARIC) Study. Arterioscler Thromb Vasc Biol. 2015;35:2700-6.

11. Perk J, De Backer G, Gohlke H, Graham I, Reiner Z, Verschuren M, et al. European guidelines on cardiovascular disease prevention in clinical practice (version 2012). The Fifth Joint Task Force of the European Society of Cardiology and Other Societies on Cardiovascular Disease Prevention in Clinical Practice (constituted by representatives of nine societies and by invited experts). Eur Heart J. 2012;33:1635-701.

12. Bournat JC, Brown CW. Mitochondrial dysfunction in obesity. Curr Opin Endocrinol Diabetes Obes. 2010;17:446-52.

13. Zamora-Mendoza R, Rosas-Vargas H, Ramos-Cervantes MT, Garcia-Zuniga P, Perez-Lorenzana H, Mendoza-Lorenzo P, et al. Dysregulation of mitochondrial function and biogenesis modulators in adipose tissue of obese children. International Journal of Obesity. 2018;42:618-24.

14. Brown DA, Perry JB, Allen ME, Sabbah HN, Stauffer BL, Shaikh SR, et al. Expert consensus document: mitochondrial function as a therapeutic target in heart failure. Nature Reviews Cardiology. 2017;14:238-50.

15. Zharikov S, Shiva S. Platelet mitochondrial function: from regulation of thrombosis to biomarker of disease. Biochem Soc Trans. 2013;41:118-23.

16. Badimon L, Hernández Vera R, Padró T, Vilahur G. Antithrombotic therapy in obesity. Thromb Haemost. 2013;110:681-8.

17. Shock LS, Thakkar PV, Peterson EJ, Moran RG, Taylor SM. DNA methyltransferase 1, cytosine methylation, and cytosine hydroxymethylation in mammalian mitochondria. Proc Natl Acad Sci USA. 2011:108:3630-5.

18. Byun H-M, Panni T, Motta V, Hou L, Nordio F, Apostoli P, et al. Effects of airborne pollutants on mitochondrial DNA methylation. Particle and Fibre Toxicology. 2013;10:18

19. Janssen BG, Byun H-M, Gyselaers W, Lefebvre W, Baccarelli AA, Nawrot TS. Placental mitochondrial methylation and exposure to airborne particulate matter in the early life environment: an ENVIRONAGE birth cohort study. Epigenetics. 2015;10:536-44.

20. Sun X, Vaghjiani V, Jayasekara WSN, Cain JE, St. John JC. The degree of mitochondrial DNA methylation in tumor models of glioblastoma and osteosarcoma. Clin Epigenetics. 2018;10(1):157.

21. Baccarelli AA, Byun H-M. Platelet mitochondrial DNA methylation: a potential new marker of cardiovascular disease. Clin Epigenetics. 2015;7:44

22. Horvath $\mathrm{S}$, Erhart $\mathrm{W}$, Brosch $\mathrm{M}$, Ammerpohl $\mathrm{O}$, von Schönfels W, Ahrens $\mathrm{M}$ et al. Obesity accelerates epigenetic aging of human liver. Proc Natl Acad Sci U S A. 2014;111:15538-43.

23. Kim M, Long TI, Arakawa K, Wang R, Yu MC, Laird PW. DNA methylation as a biomarker for cardiovascular disease risk. PLOS ONE. 2010;5:e9692.

24. Lind L, Ingelsson E, Sundström J, Siegbahn A, Lampa E. Methylation-based estimated biological age and cardiovascular disease. Eur J Clin Invest. 2018;48.

25. Roetker Nicholas S., Pankow James S., Bressler Jan, Morrison Alanna C Boerwinkle Eric. Prospective study of epigenetic age acceleration and incidence of cardiovascular disease outcomes in the ARIC study (Atherosclerosis Risk in Communities). Circ Genom Precis Med. 2018;11: e001937.

26. Zhang Y, Wilson R, Heiss J, Breitling LP, Saum K-U, Schöttker B, et al. DNA methylation signatures in peripheral blood strongly predict all-cause mortality. Nat Comm. 2017;8:14617.

27. Rosa-Garrido M Chapski DJ, Vondriska TM. Epigenomes in cardiovascular disease. Circ Res. 2018;122:1586-607. 
28. Zhang W, Song M, Qu J, Liu G-H. Epigenetic modifications in cardiovascular aging and diseases. Circ Res. 2018;123:773-86.

29. Anderson OS, Sant KE, Dolinoy DC. Nutrition and epigenetics: an interplay of dietary methyl donors, one-carbon metabolism and DNA methylation. J Nutr Biochem. 2012;23:853-9.

30. Gao X, Jia M, Zhang Y, Breitling LP, Brenner H. DNA methylation changes of whole blood cells in response to active smoking exposure in adults: a systematic review of DNA methylation studies. Clin Epigenetics. 2015;7:113.

31. Joehanes R, Just AC, Marioni RE, Pilling LC, Reynolds LM, Mandaviya PR, et al. Epigenetic signatures of cigarette smoking. Circ Cardiovasc Genet. 2016;9:436-47.

32. Rönn T, Volkov P, Gillberg L, Kokosar M, Perfilyev A, Jacobsen AL, et al. Impact of age, BMl and $\mathrm{HbAlc}$ levels on the genome-wide DNA methylation and mRNA expression patterns in human adipose tissue and identification of epigenetic biomarkers in blood. Hum Mol Genet. 2015;24: 3792-813.

33. Wahl S, Drong A, Lehne B, Loh M, Scott WR, Kunze S, et al. Epigenome-wide association study of body mass index, and the adverse outcomes of adiposity. Nature. 2017;541:81-6.

34. Mendelson MM, Marioni RE, Joehanes R, Liu C, Hedman ÅK, Aslibekyan S, et al. Association of body mass index with DNA methylation and gene expression in blood cells and relations to cardiometabolic disease: a Mendelian randomization approach. PLOS Medicine. 2017;14:e1002215.

35. D'Agostino RB, Vasan RS, Pencina MJ, Wolf PA, Cobain M, Massaro JM, et al. General cardiovascular risk profile for use in primary care: the Framingham Heart Study. Circulation. 2008:117:743-53.

36. Conroy RM, Pyörälä K, Fitzgerald AP, Sans S, Menotti A, De Backer G, et al. Estimation of ten-year risk of fatal cardiovascular disease in Europe: the SCORE project. Eur Heart J. 2003;24:987-1003.

37. Byun H, Colicino E, Trevisi L, Fan T, Christiani DC, Baccarelli AA. Effects of air pollution and blood mitochondrial DNA methylation on markers of heart rate variability. J Am Heart Assoc. 2016;22:5(4).

38. Breton CV, Song AY, Xiao J, Kim S-J, Mehta HH, Wan J, et al. Effects of air pollution on mitochondrial function, mitochondrial DNA methylation, and mitochondrial peptide expression. Mitochondrion. 2019:46:22-9.

39. Dostal V, Churchill MEA. Cytosine methylation of mitochondrial DNA at CpG sequences impacts transcription factor A DNA binding and transcription. Biochim Biophys Acta Gene Regul Mech. 2019;1862:598-607.

40. Xu Y, Li H, Hedmer M, Hossain MB, Tinnerberg H, Broberg K, et al. Occupational exposure to particles and mitochondrial DNA - relevance for blood pressure. Environ Health. 2017;9;16(1, 22).

41. Breton CV, Marsit CJ, Faustman E, Nadeau K, Goodrich JM, Dolinoy DC, et al. Small-magnitude effect sizes in epigenetic end points are important in children's environmental health studies: the children's environmental health and disease prevention research center's epigenetics working group. Environ Health Perspect. 2017:125:511-26.

42. FAD L, Muller CP, Turner JD. DNA methylation: conducting the orchestra from exposure to phenotype? Clin Epigenetics. 2016;6;:8:92.

43. Devall M, Roubroeks J, Mill J, Weedon M, Lunnon K. Epigenetic regulation of mitochondrial function in neurodegenerative disease: new insights from advances in genomic technologies. Neuroscience Letters. 2016;625:47-55.

44. Feng S, Xiong L, Ji Z, Cheng W, Yang H. Correlation between increased ND2 expression and demethylated displacement loop of mtDNA in colorectal cancer. Mol Med Rep. 2012;6:125-30.

45. Ghosh S, Singh KK, Sengupta S, Scaria V. Mitoepigenetics: the different shades of grey. Mitochondrion. 2015;25:60-6.

46. Bollati V, lodice S, Favero C, Angelici L, Albetti B, Cacace R, et al. Susceptibility to particle health effects, miRNA and exosomes: rationale and study protocol of the SPHERE study. BMC Public Health. 2014;14:1137.

47. EAPC. HeartScore - PMS [Internet]. 2012 [cited 2019 Feb 12]. Available from: https://heartscore.escardio.org/2012/calc.aspx?model = europelow.

48. WHO. ICD-9-CM [Internet]. 2007 [cited 2019 Feb 12]. Available from: https:// www.cdc.gov/nchs/icd/icd $9 \mathrm{~cm}$.htm.

49. Liu B, Du Q, Chen L, Fu G, Li S, Fu L, et al. CpG methylation patterns of human mitochondrial DNA. Scientific Reports. 2016;6:srep23421.

50. Lambertini L, Byun H-M. Mitochondrial epigenetics and environmental exposure. Curr Environ Health Rep. 2016;3:214-24.

\section{Publisher's Note}

Springer Nature remains neutral with regard to jurisdictional claims in published maps and institutional affiliations.

Ready to submit your research? Choose BMC and benefit from:

- fast, convenient online submission

- thorough peer review by experienced researchers in your field

- rapid publication on acceptance

- support for research data, including large and complex data types

- gold Open Access which fosters wider collaboration and increased citations

- maximum visibility for your research: over $100 \mathrm{M}$ website views per year

At BMC, research is always in progress.

Learn more biomedcentral.com/submissions 